DRUG THERAPY

\title{
Cetuximab or cisplatin in HNSCC?
}

For patients with locally advanced head and neck squamous-cell carcinoma (HNSCC), cetuximab in combination with radiotherapy is the established standard of care, based on pivotal phase III trial results demonstrating a significant overall survival improvement with minimal toxicity for this combination. Until now, however, no head-to-head comparison of radiotherapy plus either cetuximab or cisplatin (another radiosensitizer) in locally advanced HNSCC was available. Specifically, Michela Buglione and coauthors have conducted a randomized phase II trial to "test the hypothesis of a better compliance for cetuximab compared with cisplatin when combined with radiotherapy".

A total of 70 patients were randomly assigned to cetuximab or cisplatin treatment arms. Notably, the trial was underpowered owing to poor accrual; nevertheless, locoregional control, metastasis-free survival, and overall survival were similar in both arms. Toxicity differed with greater haematological, renal, and gastrointestinal adverse effects in the cisplatin arm, and more cutaneous toxicity and the requirement for nutritional support in the cetuximab arm. Serious adverse events related to treatment were more frequent with cetuximab.

As Buglione comments, "the most significant findings relate to the need for caution in the use of cetuximab. Other published reports underline the possibility of relevant grade $\geq 3$ toxicity for cetuximab-treated patients receiving concomitant radiotherapy". Importantly, this trial is the first randomized evidence showing worse compliance to cetuximab as opposed to cisplatin in this clinical setting. Future research plans include identifying possible biomarker-based patient stratification and "performing a subgroup analysis of our series to better elucidate the differences in compliance and outcome in the different clinical subgroups."

\section{Lisa Hutchinson}

ORIGINAL ARTICLE Magrini, S. M. et al. Cetuximab and radiotherapy versus cisplatin and radiotherapy for locally advanced head and neck cancer: a randomized phase Il trial. J. Clin. Oncol. doi;10.1200/JCO.2015.63.1671 